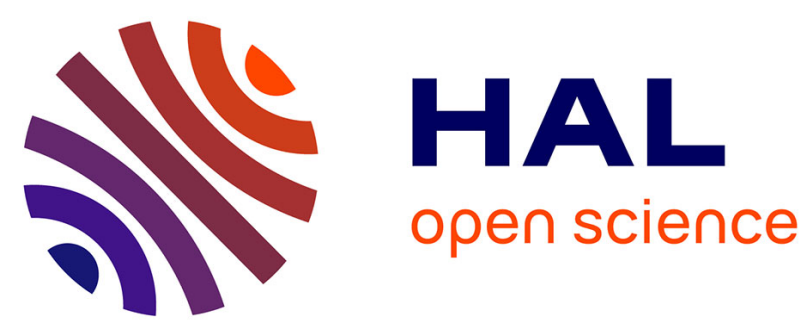

\title{
The Quasi-monochromatic ULF Wave Boundary in the Venusian Foreshock: Venus Express Observations
}

Lican Shan, Christian Mazelle, Karim Meziane, Norberto Romanelli, Yasong S. Ge, Aimin Lu, Quanming Lu, Tielong Zhang

\section{- To cite this version:}

Lican Shan, Christian Mazelle, Karim Meziane, Norberto Romanelli, Yasong S. Ge, et al.. The Quasi-monochromatic ULF Wave Boundary in the Venusian Foreshock: Venus Express Observations. Journal of Geophysical Research Space Physics, 2018, 123 (1), pp.374-384. 10.1002/2017JA024054 . insu-01673119

\section{HAL Id: insu-01673119 https://hal-insu.archives-ouvertes.fr/insu-01673119}

Submitted on 15 Sep 2020

HAL is a multi-disciplinary open access archive for the deposit and dissemination of scientific research documents, whether they are published or not. The documents may come from teaching and research institutions in France or abroad, or from public or private research centers.
L'archive ouverte pluridisciplinaire HAL, est destinée au dépôt et à la diffusion de documents scientifiques de niveau recherche, publiés ou non, émanant des établissements d'enseignement et de recherche français ou étrangers, des laboratoires publics ou privés. 


\section{Journal of Geophysical Research: Space Physics}

\section{RESEARCH ARTICLE \\ 10.1002/2017JA024054 \\ Key Points: \\ - Foreshock ULF wave boundary at \\ The Quasi-monochromatic ULF Wave Boundary in the Venusian Foreshock: Venus Express Observations} Venus is determined based on VEX observations

- The inclination angle of wave boundary increases with the IMF cone angle

- This study suggests that backstreaming ions upstream of Venus bow shock provide the main energy source of the ULF foreshock waves

Correspondence to:

L. Shan,

Icshan@mail.iggcas.ac.cn

Citation:

Shan, L., Mazelle, C., Meziane, K., Romanelli, N., Ge, Y. S., Du, A., .. Zhang, T. (2018). The quasimonochromatic ULF wave boundary in the Venusian foreshock: Venus Express observations. Journal of Geophysical Research: Space Physics, 123, 374-384. https://doi.org/10.1002/2017JA024054

Received 18 FEB 2017 Accepted 13 DEC 2017 Accepted article online 26 DEC 2017 Published online 17 JAN 2018

\author{
Lican Shan 1,2,3 D, Christian Mazelle ${ }^{4}$ iD, Karim Meziane ${ }^{5}$ iD, Norberto Romanelli,6 iD, \\ Yasong S. Ge ${ }^{1,3,7}$, Aimin $\mathrm{Du}^{1,3,7}$, Quanming $\mathrm{Lu}^{8}$ (D), and Tielong Zhang ${ }^{9}$ (iD)
}

${ }^{1}$ Key Laboratory of Earth and Planetary Physics, Institute of Geology and Geophysics, Chinese Academy of Sciences, Beijing, China, ${ }^{2}$ CAS Key Laboratory of Lunar and Deep Space Exploration, Beijing, China, Institutions of Earth Science, Chinese Academy of Sciences, Beijing, China, ${ }^{4}$ IRAP, Université de Toulouse, CNRS, UPS, CNES, Toulouse, France, ${ }^{5}$ Physics Department, University of New Brunswick, Fredericton, New Brunswick, Canada, ${ }^{6}$ LATMOS, Université Pierre et Marie Curie, Paris, France, ${ }^{7}$ College of Earth Sciences, University of Chinese Academy of Sciences, Beijing, China, ${ }^{8}$ CAS Key Lab of Geospace Environment, University of Science and Technology of China, Hefei, China, ${ }^{9}$ Space Research Institute, Austrian Academy of Sciences, Graz, Austria

Abstract The location of ultralow-frequency (ULF) quasi-monochromatic wave onset upstream of Venus bow shock is explored using Venus Express magnetic field data. We report the existence of a spatial foreshock boundary behind which ULF waves are present. We have found that the ULF wave boundary at Venus is sensitive to the interplanetary magnetic field (IMF) direction like the terrestrial one and appears well defined for a cone angle larger than $30^{\circ}$. In the Venusian foreshock, the inclination angle of the wave boundary with respect to the Sun-Venus direction increases with the IMF cone angle. We also found that for the IMF nominal direction $\left(\theta_{\mathrm{BX}}=36^{\circ}\right)$ at Venus' orbit, the value of this inclination angle is $70^{\circ}$. Moreover, we have found that the inferred velocity of an ion traveling along the ULF boundary is in a qualitative agreement with a quasi-adiabatic reflection of a portion of the solar wind at the bow shock. For an IMF nominal direction at Venus, the inferred bulk speed of ions traveling along this boundary is $1.07 V_{S W}$, sufficiently enough to overcome the solar wind convection. This strongly suggests that the backstreaming ions upstream of the Venusian bow shock provide the main energy source for the ULF waves.

\section{Introduction}

In front of a planetary bow shock, foreshock is filled with backstreaming ions and associated wave activities. The terrestrial foreshock is a rich plasma medium populated by thermal and suprathermal particles as well as ultralow-frequency (ULF) waves with various wave spectra (Eastwood et al., 2005a; Fairfield, 1969; Fuselier, 1994, 1995; Gosling et al., 1978; Hoppe et al., 1981; Paschmann et al., 1979; Russell \& Hoppe, 1983). Similar observations have also been reported at other planets such as Saturn and Mercury (e.g., Bertucci et al., 2007; Le et al., 2013), Jupiter (e.g., Bavassano-Cattaneo et al., 1987) as well as at interplanetary shocks (e.g., Blanco-Cano et al., 2016; Wilson, 2016, and references therein). Theoretical studies have found that the ULF waves result from the interaction between backstreaming ions emanating from the shock surface and the solar wind flow (e.g., Gary, 1993). Types of backstreaming ions include field-aligned beams (FABs), intermediate gyrating ions, and diffuse ion populations (e.g., Bonifazi \& Moreno, 1981; Fuselier, 1994; Gosling et al., 1978). Foreshock ion populations occupy distinct regions (Bonifazi \& Moreno, 1981), which implies the existence of boundaries between them. These boundaries are spatial frontiers between two adjacent regions characterized by distinct features (like FABs region and gyrating ions region (Mazelle et al., 2003; Meziane et al., 2004). A rapid rotation of the interplanetary magnetic field (IMF) makes the spacecraft magnetic connections before and after the rotation far from each other. This explains why in the present study the selected events are based on a small IMF monotonic rotation; otherwise the crossing of the boundary cannot be localized precisely (Andrés et al., 2015, hereafter A15). Moreover, the observed association of waves with backstreaming ions reveals the physical mechanisms of production of ion populations involved. While the FABs produced at the shock have never been observed with ULF waves, the other types of ion populations are usually associated with large-amplitude magnetic fluctuations. For instance, gyrophase-bunched ions (>3 $R_{E}$ from a bow shock) are generally observed with quasi-monochromatic ULF waves, while diffuse ions and some intermediate ions are seen with waves characterized by a wider spectrum (e.g., Fuselier et al., 1986; 
Hoppe \& Russell, 1982, 1983; Wilson, 2016; Wu et al., 2015). Theoretical (e.g., Akimoto et al., 1993; Gary, 1991; Lee, 1982; Winske \& Leroy, 1984) and observational (e.g., Eastwood et al., 2005b; Eastwood, Lucek, et al., 2005; Hobara, Walker, Balikhin, et al., 2007; Hobara, Walker, Dunlop, et al., 2007; Mazelle et al., 2003) studies offer strong evidence that $F A B s$ provide the free energy for wave excitation.

Greenstadt and Baum (1986, hereafter GB86) pioneered the first observations of wave foreshock boundary study at the terrestrial bow shock. Based on ISEE data, they reported the existence of a spatial boundary separating the region where ULF waves are present from a region where these waves are absent. The locations of the boundaries are sensitive to the IMF cone angle $\theta_{\mathrm{BX}}$ (angle between IMF line and planet-Sun direction). Using particle data, Meziane and d'Uston (1998, hereafter MD98) demonstrated the existence of an intermediate-gyrating ion boundary that coincides with the ULF boundary previously reported by GB86 when the cone angle equals to $45^{\circ}$. For the cone angle range from $20^{\circ}$ to $30^{\circ}$, the locations of boundaries differ significantly. Using Cluster data, Meziane et al. (2004) reported for the first time a spatial transition between $\mathrm{FABs}$ and gyrating ions and demonstrated that the transition coincides with the ULF compressional boundary. Using Cluster magnetic field and particle data, A15 recently revisited the terrestrial compressional ULF wave foreshock boundary. In the last study, stringent criteria were used to identify the boundary crossing. Precisely only the entries or exits of ULF waves occurring for an IMF rotation $\alpha \leq 12.5^{\circ}$ were taken into account. For this kind of analysis, this choice is dictated by the shape of the histogram of the rotation angle and not by any specific physical consideration. A smaller limit would correspond to more stationary IMF conditions but would reduce significantly the statistics. The criterion allows a precise location of the boundary crossing. The A15 study, based on the new identification of IMF angle criterion, raised more accurate results of the foreshock wave boundary for quasi-stationary IMF condition than GB86.

Studies of foreshock boundary provide insights on the physical processes occurring upstream of the bow shock. It is now established that the propagation, relative to the incident solar wind, of FABs originating from the region such as $30^{\circ}<\theta_{\mathrm{Bn}}<70^{\circ}$ (Bonifazi \& Moreno, 1981) induces electromagnetic instabilities. The excited waves in return disrupt the ion beam leading to the formation of gyrating and intermediate ions (Mazelle et al., 2000, 2003). The location where the FABs are disrupted coincides with the appearance of magnetic field fluctuations having substantial amplitude. This transition is important since it reflects the tangible effects where wave-particle interactions occur. There are several recent simulation studies about the foreshock boundary (e.g., Blanco-Cano et al., 2009; Kempf et al., 2015; Omidi et al., 2013; Savoini \& Lembège, 2015). Theoretical investigations of the foreshock ULF wave boundary are quite complex. To locate the wave boundary, Skadron et al. (1988) present a self-consistent spatiotemporal study to examine the evolution of an initial suprathermal ion population restricted in pitch angle, propagating in the solar wind plasma. Although the model is in very good agreement with observations (Meziane et al., 2004), additional studies are necessary to make an adequate assessment.

The ULF wave boundary embedded inside the Venusian foreshock is the subject of the present work. Early observations on the presence of low-frequency magnetic field fluctuations inside the Venusian foreshock were reported by Hoppe and Russell (1981), Luhmann et al. (1983), Orlowski et al. (1995), and Russell et al. (2006, and references therein). In their study of Venusian ULF waves, Orlowski et al. (1995) reported waves characterized by amplitude in the order of $\sim 1.2 \mathrm{nT}$, with a power spectrum that peaked at $0.08 \mathrm{~Hz}$ under the background magnetic field of $\sim 12 \mathrm{nT}$. They also showed that the propagation is nearly parallel to the ambient magnetic field $\left(\theta_{\mathrm{kB}} \sim 12^{\circ}\right)$. The wave properties they attained were based on a case study. An extensive work on foreshock ULF waves upstream of the Venusian bow shock was carried out recently by Shan et al. (2016). The authors restricted their study to waves that appear nearly monochromatic characterized by an unambiguous power spectrum peak. In terms of statistical average, they found that the wave period typically is equal to $27 \mathrm{~s} \pm 10 \mathrm{~s}$, while propagation angles typically are less than $30^{\circ}$ with respect to the ambient magnetic field. Both quasi-monochromatic and steepened ULF waves (e.g., shocklets) exist in planetary foreshock. An early study showed that quasi-monochromatic ULF waves are located closer to the ion foreshock boundary than the nonmonochromatic waves (Le \& Russell, 1992), which confirms a consensus that steepened waves correspond to a later phase in the wave evolution process, while highly monochromatic waves represent an early stage (Omidi \& Winske, 1990).

The existence of the terrestrial ULF foreshock boundary provides motivation to search for a similar boundary at Venus. Foreshock ULF waves at the Earth and Venus present very similar properties (Shan et al., 2016), 

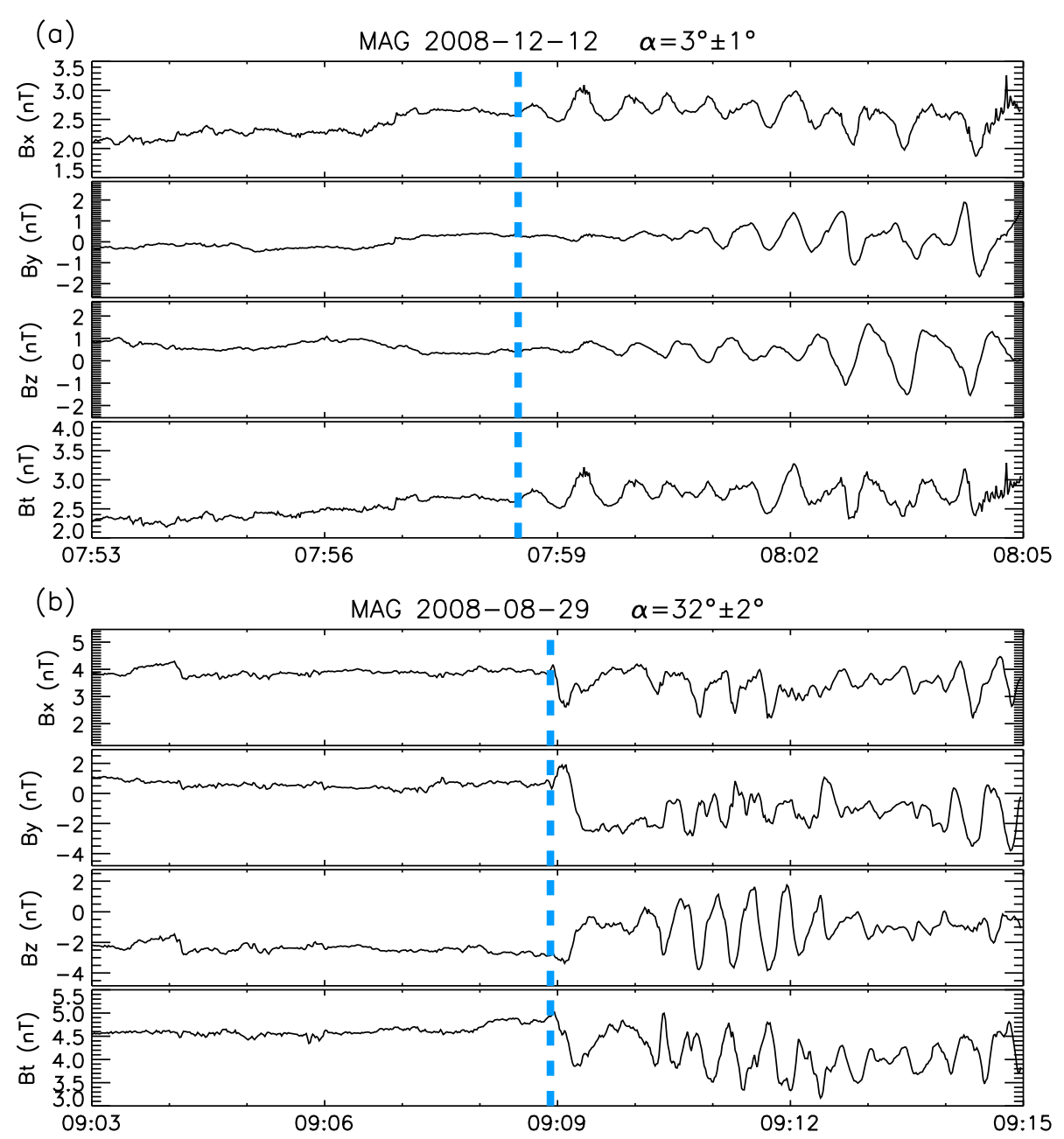

Figure 1. ULF wave foreshock boundary crossings observed by VEX with (a) $\alpha=3^{\circ} \pm 1^{\circ}$ and (b) $\alpha=32^{\circ} \pm 2^{\circ}$. The parameter $\alpha$ is the IMF rotation angle between both sides of the wave boundary. Dashed lines show the wave boundary.

although the two planets differ in terms of the global intrinsic magnetic field (since Venus has none; e.g., Luhmann, 1986). Moreover, exploring the boundary may provide a better understanding of the wave excitation mechanism. To date, the presence of backstreaming ions as a potential candidate for a free energy source to Venusian foreshock waves remains to be experimentally established. A preliminary investigation based on Pioneer Venus Orbiter magnetometer data was carried out by Greenstadt et al. (1987, hereafter G87). The authors identified entries and exits of wavelike perturbations in the IMF measurements as boundary crossings, as shown in Figure 1 of the G87 paper. Although only small changes in the IMF direction are considered in the data selection, the boundary crossing is identified based on visual inspection. For the present study, high-quality magnetic field data from Venus Express (VEX)-MAG in conjunction with stringent criteria defining a boundary crossing are employed, which are stated in detail in section 2. The results, discussion, and physical implications are given in sections 3-5, respectively. Conclusions are presented in the last section.

\section{Data Selection Methodology}

VEX was launched on 9 November 2005, providing reliable measurements on the Venusian space environment. The highly elliptical polar orbit (period of $24 \mathrm{~h}$ ) of VEX had an apogee of $\sim 11 R_{V}$ (Venus radius) upstream of the Venusian bow shock. For each orbit, the spacecraft spends $\sim 22 \mathrm{~h}$ in the solar wind and is often inside the foreshock while crossing the bow shock twice per orbital period (Zhang et al., 2008). The MAG instrument on board VEX provides $1 \mathrm{~s}$ sampling magnetic field measurements throughout the 


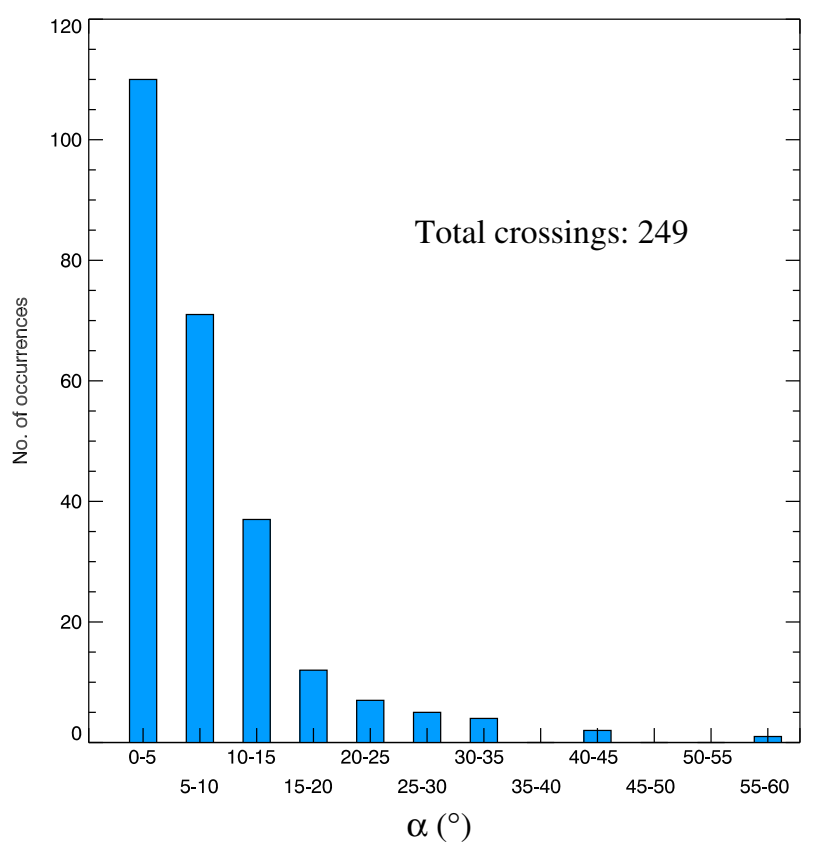

Figure 2. Histogram of the IMF rotation angle $\alpha$ associated with ULF wave boundary crossings.

full orbit (Zhang et al., 2006) for the full duration of the mission. Only MAG $1 \mathrm{~Hz}$ data are used in the present study until February 2012, and the data rate is sufficient for the study of foreshock ULF waves with a general period of 20-30 s.

The IMF line foot point connected to the bow shock that lies on the boundary separates a foreshock area into upstream and downstream regions. However, in the case when the IMF rotation with respect to the average IMF ahead of wave boundary crossing is sufficiently slow, the satellite probes very close adjacent regions and the boundary can be localized precisely. In this situation the magnetic field direction experiences a very small change across the upstream and downstream regions of the boundary. In order to select the events under quasi-stationary IMF conditions, we also calculate the ambient magnetic field direction change angle $\alpha$. The interval for calculating average field should be neither too long, thus accompanying magnetic field structure, nor too short as to create deviation because of wave phase and amplitude variations. To avoid such effects, the mean values of magnetic field are calculated from an interval of 3 times $T_{\mathrm{ULF}}$ (wave period). The angle $\alpha=\arccos \left(\mathbf{B}_{\mathbf{u}} \cdot \mathbf{B}_{\mathbf{d}} / B_{\mathrm{u}} B_{\mathrm{d}}\right)$ can be calculated between the averages of magnetic field upstream $\left(\mathbf{B}_{\mathbf{u}}\right)$ and downstream $\left(\mathbf{B}_{\mathbf{d}}\right)$ of the wave boundary. For the present study, a rotation angle upper limit of $\alpha_{C}$ (determined later) is used as a first quantitative criterion for identifying boundary crossings. Transitions to or from a wave region accompanied by IMF rotations larger than $\alpha_{\mathrm{C}}$ are not taken into account because we only concentrate on the quasi-stationary IMF condition. In addition, another criterion of qualitative nature is used for data selection included in the present work. Our study is solely restricted to the quasimonochromatic foreshock wave, and their identification criteria are illustrated in detail in a previous study (Shan et al., 2016). The peak frequency with a sufficient strong power of the wave is much less than the local proton cyclotron frequency. The frequency band near the peak power should have a very narrow range. Our choice of quasi-monochromatic waves is dictated by the reason that they correspond to a state close to the initial phase of the wave growth, and our aim is to spatially localize where these waves initiate their growth. Steepened nonlinear and proton cyclotron waves are excluded from the present study. Proton cyclotron waves, related to the hydrogen exosphere (Delva et al., 2011, 2015) commonly observed upstream of the Venusian bow shock, are not foreshock waves. From a visual perspective, a ULF boundary crossing corresponds to an appearance of a wave train onset or end.

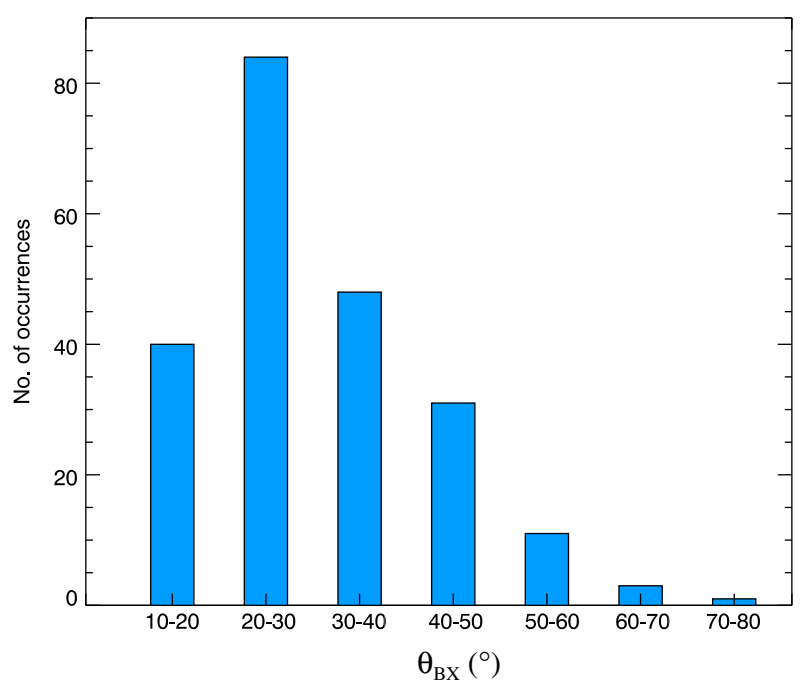

Figure 3. Histogram of IMF cone angle $\theta_{\mathrm{BX}}$ for the 218 selected events.

\section{Wave Boundary Observation}

Figure 1 depicts two events of ULF wave boundary crossings observed by VEX associated with (a) $\alpha=3^{\circ} \pm 1^{\circ}$ and (b) $\alpha=32^{\circ} \pm 2^{\circ}$, respectively. The figure shows the magnetic field components $B_{x}, B_{y}$ and $B_{z}$ in the Venus Solar Orbital (VSO) system. In the VSO coordinate system, the origin is in the center of the planet, the $X$ axis points toward the Sun, the $Y$ axis is parallel to the Venusian orbital velocity, and the $Z$ axis completes the right-hand system. The blue dashed lines mark the boundary crossings. For event (a) on 12 December 2008, VEX crossed the wave boundary at 07:58:30 UT. Downstream of the boundary, progressive and coherent ULF waves appear with moderate and gradual increase of wave amplitude. For event (b), the boundary crossing results from a rapid and large change in the IMF direction. A larger number of boundary crossings presenting signatures similar to those shown in Figure 1 have been identified. The IMF rotation angle $\alpha$ associated with each crossing is estimated. Figure 2 shows the histogram of $\alpha$ for a total of 249 ULF wave 


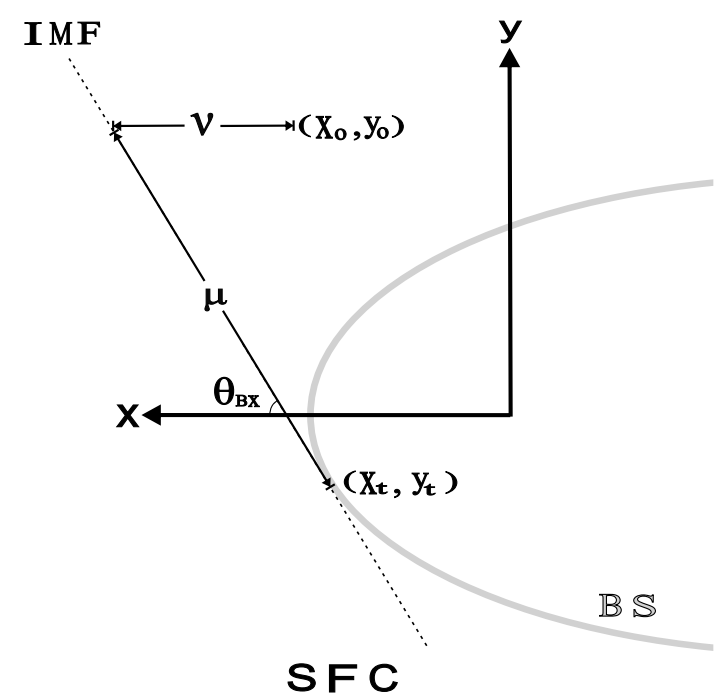

Figure 4. Schematic representation of the solar foreshock coordinates (SFC) system. The SFC frame is a cross section of the bow shock in the $B-V_{\mathrm{SW}}$ plane. Each boundary crossing is localized with the coordinates $\mu$ and $v .\left(x_{t}, y_{t}\right)$ and $\left(x_{O}, y_{O}\right)$ are the coordinates of the IMF line tangent point to the bow shock model and the observation point, respectively. Gray line represents the bow shock model. boundary crossings amid which 218 crossings are associated with $\alpha \leq 15^{\circ}$ which can be taken as the value of the critical rotation angle $\alpha_{C}$ mentioned above.

In order to explore the foreshock boundary characteristics upon the direction of the IMF, the selected boundary crossings are binned according to $\theta_{\mathrm{BX}}$ intervals. Based on our criterion, 218 wave boundary crossings are identified in the IMF cone angle range from $10^{\circ}$ to $80^{\circ}$. Figure 3 shows the distributions of IMF cone angle for the selected boundary crossings. Most of the events occur in the angle range $10^{\circ}<\theta_{\mathrm{BX}}<60^{\circ}$ with a maximum in the $20^{\circ}-30^{\circ}$ range. We only found four events for $\theta_{\mathrm{BX}}>60^{\circ}$; consequently, the statistics are not sufficient for a reliable determination of the boundary for this range. Therefore, our study is restricted for boundary crossings occurring for $0^{\circ} \leq \theta_{\mathrm{BX}} \leq 60^{\circ}$. For the selected events, the orbital and magnetic field data are transformed from VSO into an aberrated solar ecliptic system, which has an angle $4.7^{\circ}$ from VSO frame because of the Venus orbital motion ( $35 \mathrm{~km} / \mathrm{s})$. In order to explore the foreshock boundary characteristics upon the direction of the IMF, the selected boundary crossings are binned according to $\theta_{\mathrm{BX}}$ (refer to Figure 4) intervals. To allow comparison with previous studies, five $10^{\circ} \theta_{\mathrm{BX}}$ intervals respectively centered on $15^{\circ}, 25^{\circ}, 35^{\circ}, 45^{\circ}$, and $55^{\circ}$ are selected for statistical study. To determine the ULF boundary spatial location, we transformed the data from the aberrated solar ecliptic system into the solar foreshock coordinates (SFC) frame initially introduced by GB86. Figure 4 shows the schematic representation of the SFC frame. The same system of coordinates was used previously in foreshock boundary studies (MD98; Andrés et al., 2013; A15). The SFC frame is a cross section of the bow shock in the $B-V_{S W}$ plane containing the observation point. In this plane, the $X$ axis points sunward, $X-Y$ plane is the same as $B-V_{\mathrm{SW}}$ plane, and the coordinates to locate a boundary crossing are $\mu$ (distance along the tangent line) and $v$ (distance from the IMF tangent line along the Sun-planet usually called depth inside the foreshock) given by the following (shown in Figure 4):

$$
\begin{gathered}
\mu=\frac{y_{o}-y_{t}}{\sin \theta_{B X}}, \\
v=\frac{y_{o}-y_{t}}{\tan \theta_{B X}}+x_{t}-x_{o}
\end{gathered}
$$

where $\left(x_{t}, y_{t}\right)$ and $\left(x_{0}, y_{0}\right)$ are the coordinates of the IMF line tangent point to the bow shock model and the observation point, respectively. In Figure 4, the gray curve represents the model bow shock. It should be noted that since most of the waves are identified during a solar minimum activity phase, the used bow shock model is selected accordingly (Shan et al., 2015). In addition, the errors $\delta \mu$ and $\delta v$ on $\mu$ and $v$ resulting from shock model parameters uncertainty are also estimated:

$$
\delta \mu=\frac{\delta R}{\tan \theta_{B X}}, \delta v=\frac{\delta R}{\sin \theta_{B X}},
$$

where $\delta R \sim 0.248 R_{V}$ is the uncertainty which includes the variation on the standoff distance between two models and the mean of the absolute deviation. On the basis of equation (2), the errors are quite small compared with $\mu$ or $v$ when the cone angle $\theta_{\mathrm{BX}}>10^{\circ}$. In our study, there are no cases with $\theta_{\mathrm{BX}}$ less than $13^{\circ}$; therefore, the estimations of $\mu$ and $v$ are entailed with small errors.

With equation (1), we calculate the coordinates $\mu$ and $v$ for every wave boundary crossing in the SFC system. Figure 5 shows the corresponding scatterplots for fixed cone angles $\theta_{\mathrm{BX}}$ intervals: (a) $10^{\circ} \leq \theta_{\mathrm{BX}} \leq 20^{\circ}$, (b) $20^{\circ} \leq \theta_{\mathrm{BX}} \leq 30^{\circ}$, (c) $30^{\circ} \leq \theta_{\mathrm{BX}} \leq 40^{\circ}$, (d) $40^{\circ} \leq \theta_{\mathrm{BX}} \leq 50^{\circ}$, and (e) $50^{\circ} \leq \theta_{\mathrm{BX}} \leq 60^{\circ}$. On the same plot, the continuous line represents the best linear fit $(v=a \mu+b)$ for every $\theta_{\mathrm{BX}}$ range. $a$ and $b$ are the fitting slope and intercept for the wave boundary in the SFC frame. It clearly appears that the boundary is quite well defined for 

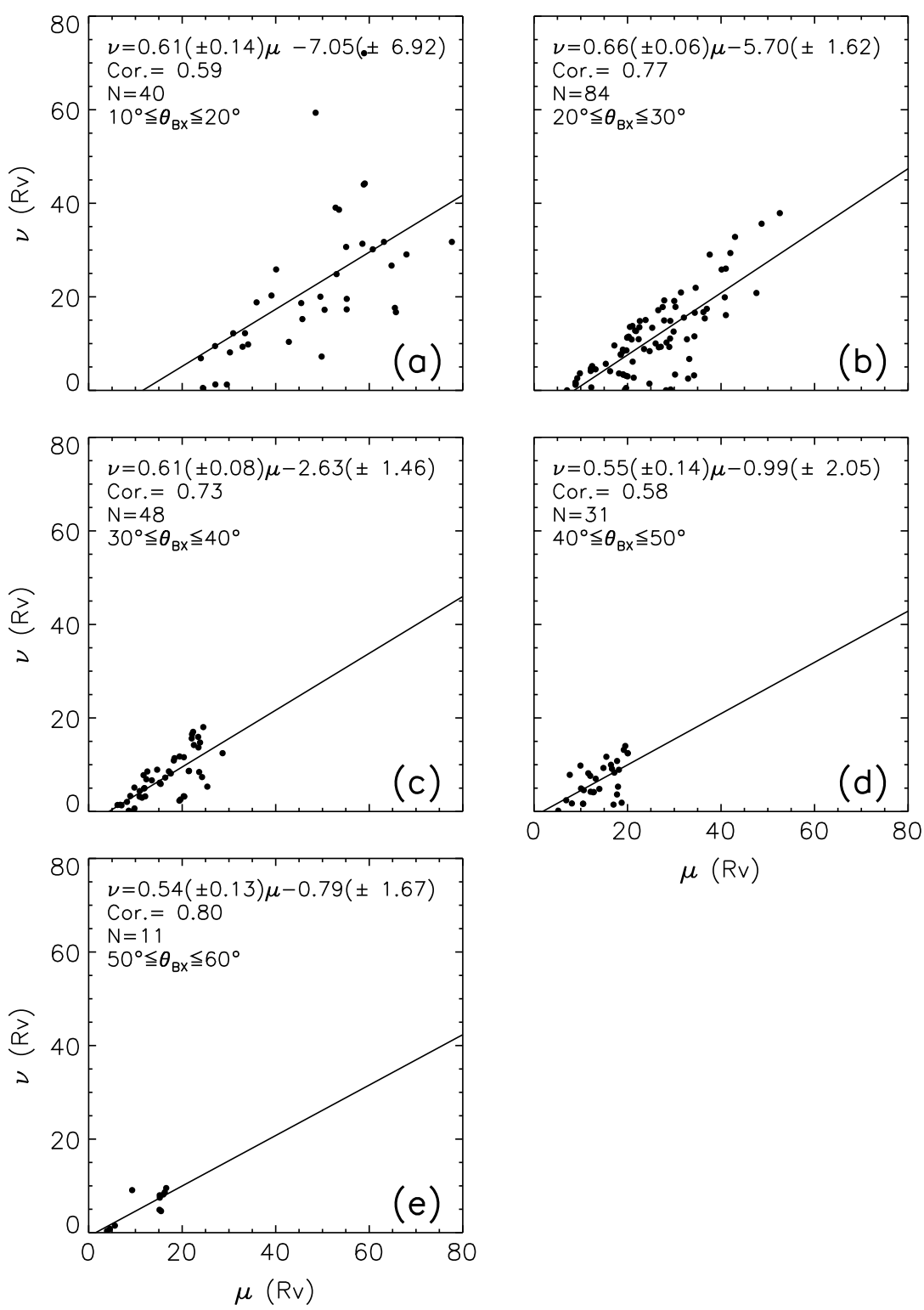

Figure 5. Scatterplots of boundary crossings for five $\theta_{\mathrm{BX}}$ ranges in SFC system. The continuous line corresponds to the best linear fit. Sample size and boundary characteristics are indicated inside each panel.

$20^{\circ} \leq \theta_{\mathrm{BX}} \leq 40^{\circ}$, the individual points approximately line up along the best fit line, which is reflected in a relatively high linear regression correlation coefficient $(>0.7)$. The correlation coefficient of the foreshock boundary location is 0.80 for $\theta_{\mathrm{BX}}>50^{\circ}$ and 0.58 for $40^{\circ} \leq \theta_{\mathrm{BX}} \leq 50^{\circ}$. The parameters of the regression line for the wave boundary at different IMF conditions are summarized in Table 1 . We also report the results

Table 1

Parameters of Regression Line $v=a \mu+b$ for the ULF Wave Boundary at Different IMF Cone Angles

\begin{tabular}{|c|c|c|c|c|c|c|c|c|c|}
\hline \multirow[b]{2}{*}{$\theta_{\mathrm{BX}}(\mathrm{deg})$} & \multicolumn{3}{|c|}{ This study } & \multicolumn{2}{|c|}{ G87 } & \multicolumn{2}{|c|}{ MD98 } & \multicolumn{2}{|c|}{ A15 } \\
\hline & $a$ & $b$ & correlation correlation & $a$ & $b$ & $a$ & $b$ & $a$ & $b$ \\
\hline $10-20$ & $0.61( \pm 0.14)$ & $-7.05( \pm 6.92)$ & 0.59 & & & & & & \\
\hline $20-30$ & $0.66( \pm 0.06)$ & $-5.70( \pm 1.62)$ & 0.77 & & & $0.56( \pm 0.02)$ & $-20.60( \pm 2.45)$ & $0.56( \pm 0.08)$ & $-6.97( \pm 5.21)$ \\
\hline $30-40$ & $0.61( \pm 0.08)$ & $-2.63( \pm 1.46)$ & 0.73 & 0.62 & -0.91 & $0.71( \pm 0.03)$ & $-20.22( \pm 1.48)$ & $0.55( \pm 0.08)$ & $-6.43( \pm 3.66)$ \\
\hline $40-50$ & $0.55( \pm 0.14)$ & $-0.99( \pm 2.05)$ & 0.58 & & & $0.64( \pm 0.02)$ & $-10.38( \pm 0.68)$ & $0.67( \pm 0.09)$ & $-8.69( \pm 3.48)$ \\
\hline $50-60$ & $0.54( \pm 0.13)$ & $-0.79( \pm 1.67)$ & 0.62 & & & $0.66( \pm 0.02)$ & $-9.23(0.61)$ & $0.77( \pm 0.10)$ & $-10.40( \pm 3.14)$ \\
\hline
\end{tabular}




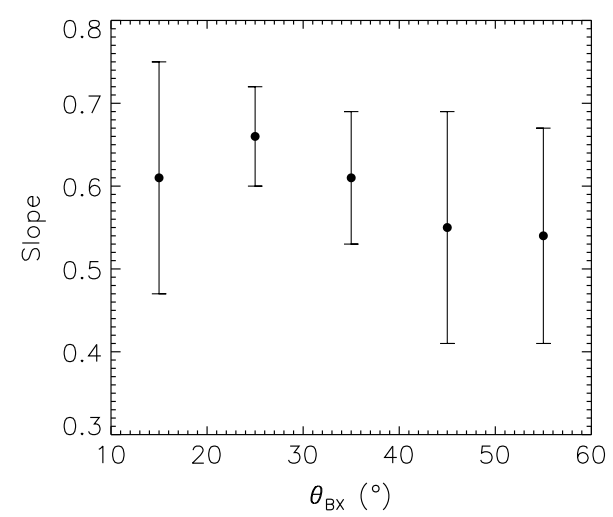

Figure 6. Variation of the boundary slope versus $\theta_{\mathrm{BX}}$. The slope and the associated error bar are obtained from the best linear fit shown in Figure 5.

from G87 (Venus), MD98 (Earth), and A15 (Earth) for comparison. It is also found that the regression line has a general trend of a decreasing slope with increasing $\theta_{\mathrm{BX}}$, except for IMF angle $10^{\circ} \leq \theta_{\mathrm{BX}} \leq 20^{\circ}$, which is also illustrated by Figure 6 . For different IMF angle range, the slopes are plotted with mean absolute deviations.

The equation of the wave boundary can also be shown in the $B-V_{\mathrm{SW}}$ plane, which is given by

$$
y=\frac{\sin \theta_{B X}}{\cos \theta_{B X}-a} x+\frac{\sin \theta_{B X}}{\cos _{B X-a}}\left(b-x_{0}\right)-y_{o},
$$

where $a$ and $b$ are the parameters from the best linear fit for each $\theta_{\mathrm{BX}}$ range shown in Figure 5.

\section{Discussion}

Using $1 \mathrm{~Hz}$ magnetic field data from VEX-MAG instrument, we investigated the existence of the foreshock boundary for quasi-monochromatic ULF waves under quasi-stationary IMF conditions. Previous studies of the ULF foreshock wave boundary did not distinguish whether these waves were quasi-monochromatic or steepened (GB86; G87; Andrés et al., 2013; A15). The present study focuses on the quasi-monochromatic ULF foreshock wave boundary. The waves are identified by the quantitative criteria described in detail in a previous study (Shan et al., 2016). Both quasi-monochromatic and steepened ULF waves (e.g., shocklets) exist in a planetary foreshock. We examined every wave event ensuring that it occurs in the foreshock region with a shock model. Based on the criteria used in this paper, a total of $218 \mathrm{ULF}$ foreshock wave boundary crossings are identified for $\theta_{\mathrm{BX}}$ from $10^{\circ}$ to $80^{\circ}$. It is also found that most of the events ( 93\%) occur in the IMF cone angle range from $10^{\circ}$ to $50^{\circ}$. Every selected event is located in the foreshock region with $\mu$ and $v$ coordinates, and we show that these coordinates are entailed with a small error when the IMF cone angle is larger than $10^{\circ}$. The nominal cone angle depends on the heliocentric distance in the Parker spiral model. For different IMF cone angles, the wave crossings have different ranges of $\mu$ and $v$. Large (small) cone angles contribute to small (large) values of $\mu$ and $v$. Based on the obtained results shown

(a)

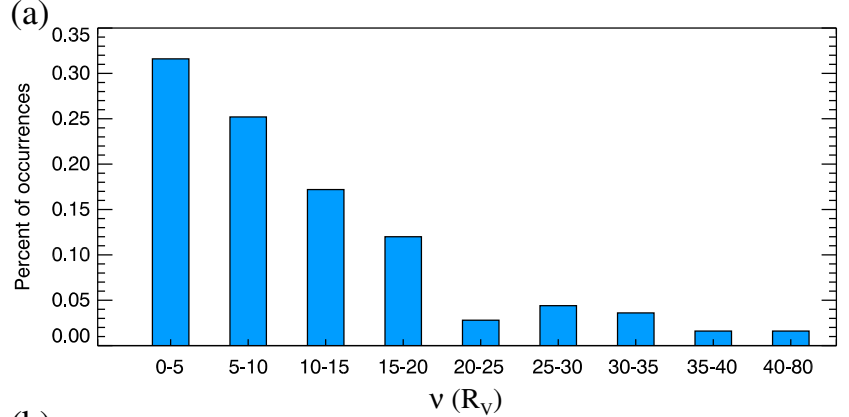

(b)

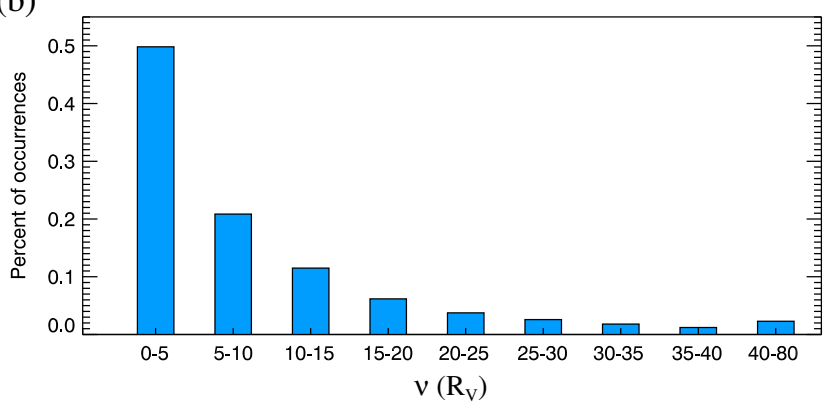

Figure 7. Histogram of $v$ for (a) quasi-monochromatic foreshock ULF waves and (b) all 10 min intervals of VEX spatial exploration in the foreshock. The location of middle point of $10 \mathrm{~min}$ interval is used for calculating the latter $v$. in Figure 5, we demonstrate that the ULF wave foreshock boundary at Venus is well defined for $20^{\circ} \leq \theta_{\mathrm{BX}} \leq 40^{\circ}$. For higher cone angle values, the boundary appears to vary more. The dependence of the boundary upon the cone angle is confirmed, a result in agreement with previous observations at the Earth (GB86; A15) and Venus (G87). For the angle range $30^{\circ} \leq \theta_{\mathrm{BX}} \leq 40^{\circ}$ (Figure $5 \mathrm{c}$ ), the slope and the intercept of the fit line for quasi-monochromatic ULF wave boundary are 0.61 and -2.63 $R_{V}$, respectively. As shown in Table 1 , taking account of uncertainties ( \pm 1.46 for intercepts) of the boundary, our results are consistent with the previous determination by G87.

Figure 7a shows the normalized distributions of foreshock depth for 218 wave boundary crossings. The total is 218 (100\%). Figure 7b shows the normalized distributions of the depth for each $10 \mathrm{~min}$ window of VEX spatial exploration in the foreshock. A total of 37,658 events (10 min interval per event) is identified in the foreshock regions. For the latter distribution (Figure $7 \mathrm{~b}$ ), the numerical value of $v$ is determined for every $10 \mathrm{~min}$ interval from all the data when VEX is in the foreshock region. Figure 7a clearly shows that most of boundary crossings ( 86\%) occur for a foreshock depth range $0<v<20 R_{V}$, while VEX quite often has a smaller depth $\left(<10 R_{V}\right)$ shown by Figure 7b. The ULF wave boundary is observed much deeper in the ion foreshock, which is consistent with foreshock knowledge when we take account of the finite growth rate of foreshock waves. 
Table 2

ULF Wave Foreshock Boundary Angle $\left(\theta_{U W F B}\right), \theta_{V n}$, and $\theta_{B n}$, for Different IMF Cone Angle Ranges

\begin{tabular}{lccc}
\hline$\theta_{\mathrm{BX}}(\mathrm{deg})$ & $\theta_{\text {UWFB }}(\mathrm{deg})$ & $\theta_{\mathrm{Bn}}(\mathrm{deg})$ & $\theta_{\mathrm{Vn}}(\mathrm{deg})$ \\
\hline $10-20$ & $36.0 \pm 21.3$ & $60.9 \pm 13.1$ & $65.8 \pm 15.5$ \\
$20-30$ & $59.7 \pm 14.4$ & $54.1 \pm 13.0$ & $65.4 \pm 14.4$ \\
$30-40$ & $69.9 \pm 13.7$ & $47.1 \pm 12.7$ & $66.4 \pm 14.3$ \\
$40-50$ & $77.4 \pm 16.6$ & $43.6 \pm 18.7$ & $61.8 \pm 11.6$ \\
$50-60$ & $87.6 \pm 14.2$ & $61.8 \pm 14.9$ & $48.9 \pm 23.7$ \\
\hline
\end{tabular}

The general decrease of the boundary slope versus the cone angle is another important result of the present study. This result contrasts with A15 findings on the terrestrial foreshock. Since Venus lacks a global magnetic field, its bow shock is much smaller as well as its associated foreshock when compared to the terrestrial foreshock (Slavin et al., 1979). It should be noted that all the events in our study occur for $\theta_{\mathrm{BX}}>13^{\circ}$, which may result in the smaller value of the slope in the $10^{\circ} \leq \theta_{\mathrm{BX}} \leq 20^{\circ}$ range than expected. In the $\mathrm{A} 15$ study, the wave boundaries have larger values of $\mu$ (with ranges $50 R_{E}<\mu<80 R_{E}$ and $20 R_{E}<\mu<50 R_{E}$ for $20^{\circ} \leq \theta_{\mathrm{BX}} \leq 30^{\circ}$ and $40^{\circ} \leq \theta_{\mathrm{BX}} \leq 50^{\circ}$, respectively; while there are $0<\mu<50 R_{V}$ and $0<\mu<20 R_{V}$ in the present study), which may result from the different spacecraft orbital coverage. As for the slope, it generally depends on the foreshock geometry and the IMF cone angle, which agrees with previous studies (GB86; Skadron et al., 1988). In addition, A15 study is not solely based on quasi-monochromatic wave train; this may also explain the main differences of the foreshock wave boundary found between the Earth and Venus. Different ranges of $\mu$ may result from the different orbital coverage.

\section{Physical Implications}

The spatial location of the foreshock wave boundary provides insights into the energy source for the ULF wave excitation, particularly that inferring to the properties of backstreaming ions. Before we inspect the link between the ULF boundary and the dynamics of backstreaming ions, it is instructive to examine the inclination of the foreshock with respect to the Venus-Sun direction. Based on equation (3), the inclination angle $\theta_{\text {UWFB }}$ (angle between wave boundary and $X$ axis) can be calculated using the numerical values of $a$ given in Table 2. As expected, we found that the inclination of the boundary increases with the cone angle. For $30^{\circ} \leq \theta_{\mathrm{BX}} \leq 40^{\circ}$ at Venus, we found that the ULF wave boundary makes an angle of $69.9^{\circ} \pm 13.7^{\circ}$. For $40^{\circ} \leq \theta_{\mathrm{BX}} \leq 50^{\circ}$, the inclination angle is $77.4^{\circ} \pm 16.6^{\circ}$, which is similar to an early result of $80^{\circ}$ (G87). At the terrestrial foreshock, $\mathrm{A} 15$ found a slope of $87.0^{\circ} \pm 6.0^{\circ}$. Compared with that at the Earth, the boundary inclination for $\theta_{\mathrm{BX}}=45^{\circ}$ is in agreement with the theoretical determination (78 $)$ of Skadron et al. (1988) study and is also in very good agreement with the outer boundary of FABs $\left(77^{\circ} \pm 3^{\circ}\right)$ (Meziane et al., 2004).

We first examine the intersection of the wave boundary location with the bow shock model. We have found that the boundary intersects the shock surface for all $\theta_{\mathrm{BX}}$ ranges considered here. For every foreshock wave boundary, we then determined the shock normal $\boldsymbol{n}$ at the intersection point and deduced the angles $\theta_{\mathrm{Vn}}$ and $\theta_{\mathrm{Bn}}$ that $\boldsymbol{n}$ makes with the solar wind and the IMF direction, respectively. We assume that the solar wind velocity is antiparallel to the $X$ axis, so $\theta_{\mathrm{Vn}}$ is between the shock normal vector and $X$ axis. The numerical values of $\theta_{\mathrm{Vn}}$ and $\theta_{\mathrm{Bn}}$ with their uncertainty are given in Table 2 . There is a trend that the shock angle $\theta_{\mathrm{Bn}}$ decreases with $\theta_{\mathrm{BX}}$ except for the range $50^{\circ} \leq \theta_{\mathrm{BX}} \leq 60^{\circ}$, which can be attributed to the poorest statistics since there are only 11 events. In all cases, it appears that the ULF boundary crosses the bow shock with a quasi-perpendicular geometry $\left(\theta_{\mathrm{Bn}} \geq 45^{\circ}\right)$, a result that is in agreement with the A15 study on the terrestrial foreshock. This result also agrees that the foreshock waves with larger IMF cone angle exist in a region with a smaller shock angle.

Following the footsteps of MD98 study, we also examine the particle momentum associated with the ULF foreshock wave boundary at Venus. The guiding center velocity $V_{\mathrm{GC}}$ of a ion traveling along the ULF wave boundary can be expressed in terms of the slope of the boundary in the $(\mu, v)$ plane. In terms of the solar wind speed $V_{\mathrm{SW}}, V_{\mathrm{GC}}=P_{\mathrm{GC}} V_{\mathrm{SW}}$ and MD98 have demonstrated that

$$
P_{\mathrm{GC}}^{2}=1-2 P \cos \theta_{\mathrm{BX}}+P^{2}
$$

where $P$ is the particle parallel velocity (normalized to the solar wind
Figure 8. The dependence of $P_{\mathrm{GC}}^{2}$ on $\cos \theta_{\mathrm{BX}}$. $P_{\mathrm{GC}}^{2}$ represents the energy of ions traveling along the wave boundary. The continuous line corresponds to the best linear fit. 
speed) expressed in the plasma rest frame of reference. The factor $P$ is related to the boundary inclination $\theta_{\text {UWFB }}$ by (MD98):

$$
P=\frac{\tan \theta_{\mathrm{UWFB}}}{\tan \theta_{\mathrm{UWFB}} \cos \theta_{\mathrm{BX}}-\sin \theta_{\mathrm{BX}}}
$$

The magnitude of $P_{\mathrm{GC}}$ for each $\theta_{\mathrm{BX}}$ can be directly evaluated from the results shown in Table 2 .

In Figure 8 we report the numerical values of $P_{\mathrm{GC}}^{2}$ versus $\cos \theta_{\mathrm{BX}}$; these values are solely based on the slope of the ULF boundary. It appears that the dependence of $P_{\mathrm{GC}}^{2}$ upon $\theta_{\mathrm{BX}}$ may be approximated by a function given by the best linear fit: $P_{\mathrm{GC}}^{2}=5.13-4.92 \cos \theta_{\mathrm{BX}}$. The qualitative agreement with expression (4) provides an estimation of the particle speed $P_{\mathrm{GC}} V_{\mathrm{SW}}$ of an ion traveling along the ULF boundary. Using the results of the linear regression and for the IMF angle $\left(\theta_{\mathrm{BX}}=36^{\circ}\right)$ (Volwerk et al., 2008), ions traveling along the boundary have a guiding center speed $V_{\mathrm{GC}}=1.07 \mathrm{~V}_{\mathrm{SW}}$.

The inferred particle velocity combined with the ULF boundary parameters allows specific insight on the production mechanism of ions susceptible to provide the energy source for the wave excitation. Based on the values obtained shown in Table 2, the specular reflection is immediately ruled out since ions produced by this mechanism escape upstream only for $\theta_{\mathrm{Bn}}$ less than $40^{\circ}$ (Schwartz et al., 1983). There is a possibility that a local curved shock or ripple (local quasi-parallel) may transform gyrating ions to FAB (Yamauchi et al., 2011). The quasi-adiabatic reflection of a portion of the solar wind (Sonnerup, 1969) is another mechanism often invoked to account for FABs present in the terrestrial foreshock. With respect to the planet reference frame, the model predicts that the particle energization $P_{\mathrm{GC}}^{2}$ is linearly dependent upon $\cos \theta_{\mathrm{BX}}$, which is in qualitative agreement with the linear fit of Figure 8. The observations pointed out that ions propagating along terrestrial ion foreshock have an average speed twice the solar wind speed that is consistent with Sonnerup's mechanism. The magnitude of $P$ derived from the ULF wave boundary above may suggest that the mechanism operating at the Venusian bow shock is similar to that at the Earth bow shock (Andrés et al., 2015). Production and acceleration of ions upstream of the Venusian bow shock have already been investigated by Yamauchi et al. (2011) using VEX observations, but they did not look into the relationships with ULF waves. A detailed case study of the evolution of the ion distributions across the ULF wave boundary is necessary to confirm the proposed scenario.

\section{Conclusion}

Using $1 \mathrm{~Hz}$ magnetic field data obtained by VEX mission, we investigated the existence of the foreshock boundary of quasi-monochromatic ULF waves upstream of Venus bow shock. The data support a welldefined spatial boundary where no ULF waves are present upstream. The location of the boundary is sensitive to the IMF cone angle and intersects the bow shock at quasi-perpendicular geometries. For a nominal direction of the IMF $\left(\theta_{\mathrm{BX}}=36^{\circ}\right)$ corresponding to the Parker spiral, the boundary makes an angle of $\sim 70^{\circ}$ with respect to the Venus-Sun direction. lons traveling along this boundary have bulk speed, estimated by the model, equal to $1.07 V_{\mathrm{SW}}$. The variation of the energy flow of particles traveling along the ULF boundary with the cone angle strongly suggests that solar wind ions reflected quasi-adiabatically at the bow shock are suitable candidates for the energy source for the wave excitation. Although Venus lacks a global magnetic field, the planet presents similar foreshock features to those seen at Earth.

The authors would like to thank the VEX MAG team and the ESA Venus Express Data Archive (psa.esac.esa.int) for providing the data. L. S. thanks C. Bertucci for his assistance in calculations. The authors are supported by the National Natural Science Foundation of China (grants 41504134, 41674168, and 41774187), the National Key Research and Development Program of China (2016YFB0501300 and 2016YFB0501304), the National Basic Research Program of China (2014CB845903), and CAS Key Laboratory of Lunar and Deep Space Exploration through grant. The authors would like to thank the reviewers for their valuable comments and suggestions towards the final version of the manuscript.

\section{References}

Akimoto, K., winske, D., Gary, S. P., \& Thomsen, M. F. (1993). Nonlinear evolution of electromagnetic ion beam instabilities. Journal of Geophysical Research, 98, 1419-1433. https://doi.org/10.1029/92JA02345

Andrés, N., Gómez, D. O., Bertucci, C., Mazelle, C., \& Dougherty, M. K. (2013). Saturn's ULF wave foreshock boundary: Cassini observations. Planetary and Space Science, 79-80, 64-75. https://doi.org/10.1016/j.pss.2013.01.014

Andrés, N., Meziane, K., Mazelle, C., Bertucci, C., \& Gómez, D. (2015). The ULF wave foreshock boundary: Cluster observations. Journal of Geophysical Research: Space Physics, 120, 4181-4193. https://doi.org/10.1002/2014JA020783

Bavassano-Cattaneo, M. B., Moreno, G., Scotto, M. T., \& Acuña, M. (1987). Observations of large-amplitude MHD waves in Jupiter's foreshock in connection with a quasi-perpendicular shock structure. Journal of Geophysical Research, 92, 12,413-12,418. https://doi.org/10.1029/ JA092iA11p12413

Bertucci, C., Achileos, N., Mazelle, C., Russell, C. T., Thomsen, M., Hospodarsky, G., ... Kurth, W. (2007). Low frequency waves in the foreshock of Saturn: First results from Cassini. Journal of Geophysical Research, 112, A09219. https://doi.org/10.1029/2006JA012098

Blanco-Cano, X., Kajdič, P., Aguilar-Rodríguez, E., Russell, C. T., Jian, L. K., \& Luhmann, J. G. (2016). Interplanetary shocks and foreshocks observed by STEREO during 2007-2010. Journal of Geophysical Research: Space Physics, 121, 992-1008. https://doi.org/10.1002/2015JA021645 
Blanco-Cano, X., Omidi, N., \& Russell, C. T. (2009). Global hybrid simulations: Foreshock waves and cavitons under radial interplanetary magnetic field geometry. Journal of Geophysical Research, 114, A01216. https://doi.org/10.1029/2008JA013406

Bonifazi, C., \& Moreno, G. (1981). Reflected and diffuse ion backstreaming from the Earth's bow shock 1. Basic properties. Journal of Geophysical Research, 86, 4397-4404. https://doi.org/10.1029/JA086iA06p04397

Delva, M., Bertucci, C., Volwerk, M., Lundin, R., Mazelle, C., \& Romanelli, N. (2015). Upstream proton cyclotron waves at Venus near solar maximum. Journal of Geophysical Research: Space Physics, 120, 344-354. https://doi.org/10.1002/2014JA020318

Delva, M., Mazelle, C., Bertucci, M. V., Vörös, Z., \& Zhang, T. L. (2011). Proton cyclotron wave generation mechanisms upstream of Venus. Journal of Geophysical Research, 116, A02318. https://doi.org/10.1029/2010JA015826

Eastwood, J. P., Balogh, A., Lucek, E. A., Mazelle, C., \& Dandouras, I. (2005a). Quasi-monochromatic ULF foreshock waves as observed by the four-spacecraft Cluster mission: 1. Statistical properties. Journal of Geophysical Research, 110, A11219. https://doi.org/10.1029/ 2004JA010617

Eastwood, J. P., Balogh, A., Lucek, E. A., Mazelle, C., \& Dandouras, I. (2005b). Quasi-monochromatic ULF foreshock waves as observed by the four-spacecraft Cluster mission: 2. Oblique propagation. Journal of Geophysical Research, 110, A11220. https://doi.org/10.1029/ 2004JA010618

Eastwood, J. P., Lucek, E. A., Narita, Y., Mazelle, C., Meziane, K., Pickett, J., ... Decreau, P. (2005). Outer magnetospheric boundaries: Cluster results. Space Science Reviews, 118(1-4), 41-94. https://doi.org/10.1007/s11214-005-3824-3

Fairfield, D. H. (1969). Bow shock associated waves observed in the far upstream interplanetary medium. Journal of Geophysical Research, 74(14), 3541-3553. https://doi.org/10.1029/JA074i014p03541

Fuselier, S. A. (1994). Suprathermal ions upstream and downstream from the Earth's bow shock. In M. J. Engebretson, K. Takahashi, \& M. Scholer (Eds.), Solar wind sources of magnetospheric ultra-low-frequency waves, Geophysical Monograph (Vol. 81, 107 p.). Washington, DC: American Geophysical Union.

Fuselier, S. A. (1995). Ion distributions in the Earth's foreshock upstream from the bow shock. Advances in Space Research, 15(8-9), 43-52. https://doi.org/10.1016/0273-1177(94)00083-D

Fuselier, S. A., Thomsen, M. F., Gosling, J. T., Bame, S. J., \& Russell, C. T. (1986). Gyrating and intermediate ion distributions upstream from the Earth's bow shock. Journal of Geophysical Research, 91, 91-99. https://doi.org/10.1029/JA091iA01p00091

Gary, S. P. (1991). Electromagnetic ion/ion instabilities and their consequences in space plasmas: A review. Sciences in Space Research, 56, 373-415.

Gary, S. P. (1993). Theory of plasma microestabilities. New York: Cambridge University Press. https://doi.org/10.1017/CBO9780511551512

Gosling, J. T., Ashridge, J. R., Bame, S. J., Paschmann, G., \& Sckopke, N. (1978). Observations of two distinct populations of bow shock ions in the upstream solar wind. Geophysical Research Letters, 5, 957-960. https://doi.org/10.1029/GL005i011p00957

Greenstadt, E., \& Baum, L. (1986). Earth's compressional foreshock boundary revisited: Observations by the ISEE 1 magnetometer. Journal of Geophysical Research, 91, 9001-9006. https://doi.org/10.1029/JA091iA08p09001

Greenstadt, E. W., Baum, L. W., Jordan, K. F., \& Russell, C. T. (1987). The compressional ULF foreshock boundary of Venus: Observations by the PVO magnetometer. Journal of Geophysical Research, 92, 3380-3384. https://doi.org/10.1029/JA092iA04p03380

Hobara, Y., Walker, S. N., Balikhin, M., Pokhotelov, O. A., Dunlop, M., Nilsson, H., \& Rème, H. (2007). Characteristics of terrestrial foreshock ULF waves: Cluster observations. Journal of Geophysical Research, 112, A07202. https://doi.org/10.1029/2006JA012142

Hobara, Y., Walker, S. N., Dunlop, M., Balikhin, M., Pokhotelov, O. A., Nilsson, H., \& Réme, H. (2007). Mode identification of terrestrial ULF waves observed by Cluster: A case study. Planetary and Space Science, 55(15), 2257-2260. https://doi.org/10.1016/j.pss.2007.05.020

Hoppe, M. M., \& Russell, C. T. (1981). On the nature of ULF waves upstream of planetary bow shocks. Advances in Space Research, 1(1), 327-332. https://doi.org/10.1016/0273-1177(81)90129-0

Hoppe, M. M., \& Russell, C. T. (1982). Particle acceleration at planetary bow shock waves. Nature, 295(5844), 41-42. https://doi.org/10.1038/ $295041 \mathrm{a} 0$

Hoppe, M. M., \& Russell, C. T. (1983). Plasma rest frame frequencies and polarizations of the low-frequency upstream waves: ISEE 1 and 2 observations. Journal of Geophysical Research, 88, 2021-2028. https://doi.org/10.1029/JA088iA03p02021

Hoppe, M. M., Russell, C. T., Frank, L. A., Eastman, T. E., \& Greenstadt, E. W. (1981). Upstream hydromagnetic waves and their association with backstreaming ion populations: ISEE-1 and -2 observations. Journal of Geophysical Research, 86, 4471-4492. https://doi.org/10.1029/ JA086iA06p04471

Kempf, Y., Pokhotelov, D., Gutynska, O., Wilson III, L. B., Walsh, B. M., Alfthan, S., ... Palmroth, M. (2015). Ion distributions in the Earth's foreshock: Hybrid-Vlasov simulation and THEMIS observations. Journal of Geophysical Research: Space Physics, 120, 3684-3701. https://doi.org/ 10.1002/2014JA020519

Le, G., Chi, P. J., Blanco-Cano, X., Boardsen, S., Slavin, J. A., \& Anderson, B. J. (2013). Upstream ultra-low frequency waves in Mercury's foreshock region: MESSENGER magnetic field observations. Journal of Geophysical Research: Space Physics, 118, 2809-2823. https://doi.org/ $10.1002 /$ jgra. 50342

Le, G., \& Russell, C. T. (1992). A study of ULF wave foreshock morphology-II: Spatial variation of ULF waves. Planetary and Space Science, 40(9), 1215-1225. https://doi.org/10.1016/0032-0633(92)90078-3

Lee, M. A. (1982). Coupled hydromagnetic waves excitation and ion acceleration upstream of the Earth's bow shock. Journal of Geophysical Research: Space Physics, 87, 5063-5080.

Luhmann, J. G. (1986). The solar wind interaction with Venus. Space Science Reviews, 44, 241-306.

Luhmann, J. G., Tatrallyay, M., Russell, C. T., \& Winterhalter, D. (1983). Magnetic field fluctuations in the Venus magnetosheath. Geophysical Research Letters, 10, 655-658. https://doi.org/10.1029/GL010i008p00655

Mazelle, C., Le Quéau, D., \& Meziane, K. (2000). Nonlinear wave-particle interaction upstream from the Earth's bow shock. Nonlinear Processes in Geophysics, 7, 185-190.

Mazelle, C., Meziane, K., LeQuéau, D., Wilber, M., Eastwood, J. P., Rème, H., ... Balogh, A. (2003). Production of gyrating ions from nonlinear wave-particle interaction upstream from the Earth's bow shock: A case study from Cluster-CIS. Planetary and Space Science, 51(12), 785-795. https://doi.org/10.1016/j.pss.2003.05.002

Meziane, K., \& d'Uston, C. (1998). A statistical study of the upstream intermediate ion boundary in the Earth's foreshock. Annales Geophysicae 16(2), 125-133. https://doi.org/10.1007/s005850050585

Meziane, K., Wilber, M., Mazelle, C., LeQuéau, D., Kucharek, H., Lucek, E. A., ... Lundin, R. N. (2004). Simultaneous observations of fieldaligned beams and gyrating ions in the terrestrial foreshock. Journal of Geophysical Research, 109, A05107. https://doi.org/10.1029/ 2003JA010374

Omidi, N., \& Winske, D. (1990). Steepening of kinetic magnetosonic waves into shocklets: Simulations and consequences for planetary shocks and comets. Journal of Geophysical Research, 95, 2281-2300. https://doi.org/10.1029/JA095iA03p02281 
Omidi, N., Zhang, H., Sibeck, D., \& Turner, D. (2013). Spontaneous hot flow anomalies at quasi-parallel shocks: 2. Hybrid simulations. Journal of Geophysical Research: Space Physics, 118, 173-180. https://doi.org/10.1029/2012JA018099

Orlowski, D. S., Russell, C. T., Krauss-Varban, D., \& Omidi, N. (1995). Properties of ultralow frequency upstream waves at Venus and Saturn: A comparison. Advances in Space Research, 16(4), 143-148. https://doi.org/10.1016/0273-1177(95)00221-Y

Paschmann, G., Sckopke, N., Bame, S. J., Asbridge, J. R., Russell, C. T., \& Greenstadt, E. W. (1979). Association of low-frequency waves with suprathermal ions in the upstream solar wind. Geophysical Research Letters, 6, 209-212. https://doi.org/10.1029/GL006i003p00209

Russell, C. T., \& Hoppe, M. M. (1983). Upstream waves and particles. Space Science Reviews, 34, 155-172.

Russell, C. T., Luhmann, J. G., \& Strangeway, R. J. (2006). The solar wind interaction with Venus through the eyes of the Pioneer Venus Orbiter. Planetary and Space Science, 54(13-14), 1482-1495. https://doi.org/10.1016/j.pss.2006.04.025

Savoini, P., \& Lembège, B. (2015). Production of nongyrotropic and gyrotropic backstreaming ion distributions in the quasi-perpendicular ion foreshock region. Journal of Geophysical Research: Space Physics, 120, 7154-7171. https://doi.org/10.1002/2015JA021018

Schwartz, S. J., Michelle, F., Thomsen, F., \& Gosling, J. T. (1983). lons upstream of the Earth's bow shock: A theoretical comparison of alternative source populations. Journal of Geophysical Research, 88, 2039-2047. https://doi.org/10.1029/JA088iA03p02039

Shan, L., Lu, Q., Mazelle, C., Huang, C., Zhang, T., Wu, M., ... Wang, S. (2015). The shape of the Venusian bow shock at solar minimum and maximum: Revisit based on VEX observations. Planetary and Space Science, 109-110, 32-37. https://doi.org/10.1016/j.pss.2015.01.004

Shan, L., Mazelle, C., Meziane, K., Delva, M., Lu, Q., Ge, Y. S., ... Zhang, T. (2016). Characteristics of quasi-monochromatic ULF waves in the Venusian foreshock. Journal of Geophysical Research: Space Physics, 121, 7385-7397. https://doi.org/10.1002/2016JA022876

Skadron, G., Holdaway, R. D., \& Lee, M. A. (1988). Formation of the wave compressional boundary in the Earth's foreshock. Journal of Geophysical Research, 93, 11,354-11,362. https://doi.org/10.1029/JA093iA10p11354

Slavin, J. A., Elphic, R. C., \& Russell, C. T. (1979). A comparison of Pioneer Venus and Venera bow shock observations: Evidence for a solar cycle variation. Geophysical Research Letters, 6, 905-908. https://doi.org/10.1029/GL006i011p00905

Sonnerup, B. U. Ö. (1969). Acceleration of particles reflected at a shock front. Journal of Geophysical Research, 74, 1301-1304. https://doi.org/ 10.1029/JA074i005p01301

Volwerk, M., Zhang, T. L., Delva, M., Vörös, Z., Baumjohann, W., \& Glassmeier, K.-H. (2008). Mirror-mode-like structures in Venus' induced magnetosphere. Journal of Geophysical Research, 113, E00B16. https://doi.org/10.1029/2008JE003154

Wilson, L. B. III (2016). Low-frequency waves at and upstream of collisionless shocks. In A. Keiling, D. -H. Lee, \& V. Nakariakov (Eds.), Lowfrequency waves in space plasmas, Geophysical Monograph Series (Vol. 216, pp. 269-291). Washington, DC: American Geophysical Union. https://doi.org/10.1002/9781119055006.ch16

Winske, D., \& Leroy, M. M. (1984). Diffused ions produced by ion beam instabilities. Journal of Geophysical Research, 89, 2673-2688. https:// doi.org/10.1029/JA089iA05p02673

Wu, M., Hao, Y., Lu, Q., Huang, C., Guo, F., \& Wang, S. (2015). The role of large amplitude upstream low-frequency waves in the generation of superthermal ions at a quasi-parallel collisionless shock: Cluster observations. The Astrophysical Journal, 808(1), 2. https://doi.org/10.1088/ 0004-637X/808/1/2

Yamauchi, M., Futaana, Y., Fedorov, A., Frahm, R. A., Winningham, J. D., Dubinin, E., ... Fraenz, M. (2011). Comparison of accelerated ion populations observed upstream of the bow shocks at Venus and Mars. Annales Geophysicae, 29(3), 511-528. https://doi.org/10.5194/ angeo-29-511-2011

Zhang, T. L., Baumjohann, W., Delva, M., Auster, H. U., Balogh, A., Russell, C. T., ... Lebreton, J. P. (2006). Magnetic field investigation of the Venus plasma environment: Expected new results from Venus Express. Planetary and Space Science, 54(13-14), 1336-1343. https://doi.org/ 10.1016/j.pss.2006.04.018

Zhang, T. L., Delva, M., Baumjohann, W., Volwerk, M., Russell, C. T., Barabash, S., ... Zambelli, W. (2008). Initial Venus Express magnetic field observations of the Venus bow shock location at solar minimum. Planetary and Space Science, 56(6), 785-789. https://doi.org/10.1016/j. pss.2007.09.012 